\title{
A Idade como Diferencial no Engagement dos Profissionais de Enfermagem
}

\author{
Keiti Garbin ${ }^{1}$ (D, Adriano Pasqualotti ${ }^{1}$, Maria José Chambel $^{2}$, \& Cleide Fátima Moretto \\ ' Universidade de Passo Fundo, Passo Fundo, RS, Brasil \\ ${ }^{2}$ Universidade de Lisboa, Lisboa, Portugal
}

\begin{abstract}
RESUMO - O engajamento no trabalho é definido pelo equilíbrio entre funções cognitiva e afetiva do profissional. O estudo objetiva identificar a relação entre o engajamento e a idade dos profissionais de enfermagem, em termos de vigor, dedicação e absorção, atuantes em diferentes organizações de saúde. Com corte transversal, nível de complexidade descritiva, abordagem quantitativa, contempla 900 profissionais da enfermagem inscritos no Coren-RS, Brasil. O instrumento de pesquisa abrange questões sociodemográficas e o questionário breve de engajamento no trabalho de Utrecht. Os resultados apontam um nível médio de engajamento, especialmente no atributo relacionado à dedicação. Níveis mais elevados de engajamento foram identificados em profissionais com até 34 anos e com mais de 40 anos de idade.
\end{abstract}

PALAVRAS-CHAVE: engajamento, enfermagem, saúde do trabalhador

\section{The Age as a Differential in The Engagement of Nursing Professionals}

\begin{abstract}
Work engagement is defined by a balance between the cognitive and affective part of the professional. This study aims to identify the relationship between the engagement and the age of nursing professionals, in terms of vigor, dedication and absorption, acting in different health organizations. The cross-sectional study, with descriptive complexity level, quantitative approach, includes 900 nursing professionals enrolled in the Coren-RS, Brazil. The research instrument covers sociodemographic issues and the brief engagement questionnaire in Utrecht's work. The results indicate an average level of engagement, specially for the attribute related to dedication. Higher engagement levels were identified in professionals up to 34 years and over 40 years of age.
\end{abstract}

KEYWORDS: engagement, nursing, worker's health

Há um consenso de que os aspectos psicossociais do trabalho são determinantes cruciais da saúde (International Labour Organization [ILO], 2019). Os recursos laborais oferecidos por parte das empresas contribuem para a promoção do bem-estar e da qualidade de vida dos trabalhadores. Estes recursos, a realização de metas de trabalho ou mesmo a satisfação de necessidades básicas, estimulam um estado de espírito positivo relacionado ao trabalho. Esse estado afetivo-motivacional promove resultados organizacionais positivos, como comprometimento e desempenho organizacional.
Neste contexto, assume-se que o engajamento (engagement) no trabalho intermedia a relação entre os recursos profissionais e os resultados organizacionais (Schaufeli \& Taris, 2016). O engajamento no trabalho é um multiconceito motivacional dimensional, que demostra, simultaneamente, o investimento do físico de uma pessoa, a energia emocional e cognitiva em trabalho ativo e desempenho (Rich, Lepine, \& Crawford, 2010).

William Kahn dedicou seus estudos sobre as condições psicológicas pelas quais as pessoas pessoalmente se engajam ou desengajam no trabalho (Kahn, 1990). Explicou que

*E-mail: keitig1@hotmail.com

- Submetido: 07/07/2019; Revisado: 29/11/2019; Aceito: 03/12/2019. 
estas condições se constituem em experiências psicológicas de elementos racionais e inconscientes dos contextos de trabalho. Defendeu que os contextos de trabalho, mediados pelas percepções das pessoas, criam condições pelas quais ocorre o engajamento ou o desengajamento.

O engajamento tem sido conceituado como um estado mental satisfatório, persistente e positivo em relação às atividades laborais, composto pelas dimensões vigor, dedicação e absorção (Borges, Abreu, Queirós, \& Maio, 2017). O vigor está associado aos níveis elevados de energia e grande resiliência no trabalho, podendo assim superar dificuldades. A dedicação se refere à forma pela qual o trabalhador se envolve nas suas atividades laborais, bem como sua inspiração e entusiasmo em relação a ele. A absorção, por sua vez, está associada à concentração evidenciada pelo profissional nas atividades, ficando tão envolvido a ponto de não notar o tempo passar.

No âmbito da psicologia positiva, nota-se que os estudos sobre engagement e Burnout devem ser medidos independentemente, com instrumentos distintos por serem considerados conceitos opostos (Demerouti, Mostert, \& Bakker, 2010). O engajamento no trabalho é definido por um equilíbrio entre a parte cognitiva e afetiva do profissional, com trabalhadores apresentam-se motivados, proativos e adaptam-se com facilidade aos diferentes contextos (Pocinho \& Perestrelo, 2011). Burnout e engagement são o resultado das vivências no ambiente de trabalho partindo da combinação entre as exigências do processo de trabalho e os recursos viáveis para lidar com essas demandas tais como condições psicológicas de motivação e drenagem energética (Obregon et al., 2016). A síndrome de Burnout, por sua vez, é uma resposta da cronificação ao estresse ocupacional no meio laboral (Barboza \& Baresin, 2007; Ferreira \& Lucca, 2015; Rolim, 2013), caracterizada como uma síndrome multifatorial, composta pela exaustão emocional, despersonalização ou cinismo, e reduzida realização profissional.

A identificação e o envolvimento das pessoas são pontos essenciais na formação de uma equipe coesa e com energia em um ambiente de grande competitividade (Martins, Costa, \& Siqueira, 2015). De outra parte, as empresas priorizam trabalhadores que tenham capacidade física e mental que os possibilite superar e lidar com as demandas, adversidades e conflitos no ambiente organizacional (Vicencio-Ayub, Jurado-Cárdenas, \& Valencia-Cruz, 2015). Profissionais engajados mobilizam mais recursos tanto psicológicos quanto de trabalho, motivo pelo qual justifica a importância de que o espaço de trabalho promova bem-estar e qualidade de vida (Freitas \& Charão-Brito, 2016).

Os profissionais da assistência costumam ser os mais atingidos pelo Burnout, devido ao próprio contexto da profissão, uma vez que estão em contato constante com pacientes e familiares em situações de vulnerabilidade (Barboza \& Baresin, 2007). Entre as profissões mais desgastantes, é possível destacar os trabalhadores da enfermagem. Estes trabalhadores estão expostos a fatores físicos, químicos, biológicos e psíquicos, além da ausência de reconhecimento no cotidiano e das prolongadas e aceleradas jornadas de trabalho (Hanzelmann \& Passos, 2010).

Neste contexto, o ambiente de trabalho e as características pessoais, além da bagagem adquirida ao longo da carreira profissional, são vistos como fatores decisivos para o engajamento no trabalho da enfermagem (Borges et al., 2017). São considerados preditores do engajamento a confiança nos colegas, na organização onde exercem funções, e nas chefias e supervisores (Gonçalves, Galvão, Escanciano, Pinheiro, \& Gomes, 2018), além da autonomia na profissão. A complexidade prática em obter a delimitação dos papéis entre enfermeiros, técnicos e auxiliares de enfermagem acaba por acentuar a questão da autonomia (Ferreira \& Lucca, 2015).

Recentes estatísticas do trabalho em nível internacional demostram que a idade média da força de trabalho vem aumentando, mostrando uma tendência a um perfil de força de trabalho mais velha em todo o mundo (Ng \& Feldman, 2010). Devido ao crescente envelhecimento da força de trabalho, pesquisadores e gestores das organizações concentram sua atenção nos profissionais mais maduros, isto é, nas suas expectativas, no seu desempenho e nas suas necessidades (Kooij, Lange, Jansen, Kanfer \& Dikkers, 2011). Neste contexto, ganham relevância para os gestores, concomitantemente, o envelhecimento da força de trabalho e as mudanças em termos do seu engajamento (Avery, Mcfay, \& Wilson, 2007).

No âmbito da psicologia ocupacional, o modelo job demands-resources (Demerouti et al., 2001), ou modelo de exigências-recursos laborais, propõe que o bem-estar, observado por meio de baixo Burnout e estresse e altos níveis de engajamento, responde ao equilíbrio entre as demandas do trabalho e os recursos disponíveis para a sua execução. Os estudos dele decorrentes tem evidenciado resultados não significativos entre diferentes grupos de idade (Ramos, Jenny, \& Bauer, 2016). Tacitamente, assume-se que as exigências do trabalho e os recursos profissionais são tomados como constantes ao longo do ciclo de vida. Poucos estudos têm se voltado para a relação entre a idade e o engajamento. Enquanto de uma parte os trabalhadores mais velhos poderiam estar menos engajados em virtude da proximidade com a aposentadoria, por outra, contam com segurança do emprego e pagamentos crescentes, o que os tornaria mais engajados em relação aos trabalhadores mais jovens (Salas-Vallina \& Alegre, 2017).

Nesta perspectiva, o artigo tem como objetivo identificar a relação entre o engajamento e a idade dos profissionais de enfermagem, em termos de vigor, dedicação e absorção, que atuam em diferentes organizações de saúde. Busca-se avaliar, ainda, possíveis associações entre tal relação e outras características sociodemográficas, a exemplo do enquadramento profissional, do gênero, da escolarização, do tempo de atuação e do vínculo funcional. 


\section{MÉTODO}

Trata-se de um estudo de corte transversal com profissionais da enfermagem inscritos no Conselho Regional de Enfermagem no estado do Rio Grande do Sul (Coren-RS). No momento da pesquisa, o Coren-RS possuía 122.763 inscritos em outubro de 2018 (Cofen, 2018). A população do estudo compreende os profissionais da enfermagem registrados no referido conselho, de ambos os sexos, com mais de 18 anos de idade, que trabalham em organizações de saúde, públicas e privadas por um período superior a seis meses consecutivos na enfermagem durante o período de coleta de dados. Após o consentimento do Coren-RS, foi emitida nota sobre a pesquisa no website da entidade e enviada mensagem eletrônica a cada profissional registrado, informando sobre a pesquisa e indicando o local de acesso ao instrumento de pesquisa. Portanto, a intenção foi acessar todos os profissionais registrados, na perspectiva de obter uma amostragem representativa. A coleta de dados foi realizada entre os meses de fevereiro e abril de 2018. O estudo foi aprovado pelo Comitê de Ética em Pesquisa da Universidade de Passo Fundo (Parecer $n^{\circ}$ 2.465.770).

Participaram do estudo 900 profissionais da enfermagem do estado do Rio Grande do Sul, que concordaram com o Termo de Consentimento Livre e Esclarecido (TCLE). Foram seguidas as diretrizes da Resolução CNS n ${ }^{\circ}$ 466/12 do Conselho Nacional de Saúde sobre a participação de pessoas em pesquisas, bem como foram atendidos os aspectos éticos de consentimento do Coren-RS, de sigilo, anonimato e respeito aos valores do sujeito. Foram excluídos do estudo profissionais da enfermagem com menos de seis meses consecutivos de trabalho na área da enfermagem e os indivíduos cujas informações não foram preenchidas corretamente ou estavam incompletas no instrumento proposto pelos autores neste trabalho. Foram identificados indícios de validade para a escala Engagement por meio de medidas de adequação à amostra da estrutura fatorial que apresentou o número de fatores comumente apresentados pelo instrumento. A avaliação da validade de constructo ocorreu pelo método de Componentes Principais, pelo teste de confiabilidade Alfa de Cronbach, além da normatização obtida pelas estatísticas descritivas dos constructos resultantes (Pestana \& Gageiro, 2000). Em função dos resultados análise dos componentes principais, procedeu-te às estimativas do teste $\mathrm{t}$ de Student e análise de variância (Anova), com comparações múltiplas, e a aplicação do teste de Tukey HSD.

Para conhecer o perfil sociodemográfico e as condições que podem influenciar os resultados da percepção dos participantes no que diz respeito ao engajamento no trabalho, foram analisadas variáveis associadas à formação profissional, sexo, idade, escolaridade, número de vínculos empregatícios, condição na unidade familiar, se possui outro emprego em área diferente da enfermagem, carga horária semanal total, turno de trabalho, renda bruta mensal, tipo de instituição de saúde que trabalha, tempo de trabalho na enfermagem. Para avaliar o engajamento foi utilizado o questionário Breve de Engagement no Trabalho de Utrecht (Schaufeli, Bakker, \& Salanova, 2006), que abrange nove itens. Os participantes responderam a cada um desses itens por meio de uma escala ordinal de 7 pontos ( 0 para nunca, 6 para todos os dias).

Os dados quantitativos foram analisados por meio de análise estatística descritiva, teste t de Student, teste de quiquadrado e Análise de Variância - ANOVA, no IBM SPSS Statistics 24, para um nível de significância de p $<0,05$. Utilizou-se o coeficiente Alfa ( $\alpha$ ) de Cronbach que resultou em um valor de 0,921 , indicando $63 \%$ de consistência entre as variáveis. A determinação dos fatores foi realizada por meio da técnica de componentes principais.

\section{RESULTADOS E DISCUSSÃO}

A partir dos critérios de inclusão e exclusão do estudo, participaram da amostra 900 profissionais da enfermagem. Como pode-se observar pela Tabela 1 , em termos do enquadramento profissional, $442(49,1 \%)$ participantes são enfermeiros, $421(46,8 \%)$ técnicos de enfermagem, 30 $(3,3 \%)$ auxiliares ou atendentes de enfermagem e $7(0,8 \%)$ exercem outra atividade. Predominam profissionais do sexo feminino, $795(88,3 \%)$ e a idade a média foi de 38,11 anos, confirmando os resultados apontados pela literatura (Hanzelmann \& Passos, 2010).

A participação expressiva de Enfermeiros também está associada ao nível mais alto de escolarização: $57,5 \%$ dos participantes possuem o ensino superior completo $(17,2 \%)$ e pós-graduação (40,3\%), em nível de especialização, de mestrado ou de doutorado. Em termos da condição de provisão da renda na unidade familiar, destaca-se a condição de contribuinte parcial $(59,9 \%)$, associada à renda complementar, típico na condição do gênero feminino no mercado de trabalho. A maior parte dos participantes do estudo, todavia, está vinculado a um único emprego ou trabalho $(80,6 \%)$, predominantemente formal $(75,9 \%)$, em diferentes instituições. No que diz respeito ao tempo de atuação profissional, a média foi de 12,8 anos, com dados indicando que $40,2 \%$ dos entrevistados atuam na profissão entre 8 e 14 anos, com predomínio de uma carga horária de 40 horas semanais $(42,2 \%)$, evidências próximas aos resultados de França et al. (2012). Em relação à instituição de vínculo dos participantes, sobressaem-se as situações 
de mais de uma instituição ou outra (34,4\%). Os locais de atuação são variados, sendo que $25,3 \%$ exercem a atividade em setores fechados ou emergência.

$\mathrm{Na}$ análise específica do nível de engagement (Tabela 2) foi identificado um índice de 4,96 $(\mathrm{DP}=1,50)$ que pode ser considerado relativamente elevado. A mediana foi de 5,22. Em relação aos itens de avaliação nas questões propostas, os escores mais elevados estão relacionados à dedicação, como o item que refere o sentimento de orgulho em relação ao trabalho realizado $(6,10)$. Já a menor média $(4,47)$ foi

Tabela 1

Características gerais dos participantes da pesquisa

\begin{tabular}{|c|c|c|c|}
\hline Variável & Atributos & Frequência absoluta & Frequência relativa $(\%)$ \\
\hline \multirow[t]{4}{*}{ Enquadramento } & Enfermeiro (a) & 442 & 49,1 \\
\hline & Técnico (a) em Enfermagem & 421 & 46,8 \\
\hline & Auxiliar/Atendente em Enfermagem & 30 & 3,3 \\
\hline & Outra & 7 & 0,8 \\
\hline \multirow[t]{3}{*}{ Sexo } & Feminino & 795 & 88,3 \\
\hline & Masculino & 102 & 11,3 \\
\hline & Preferiu não informar & 3 & 0,3 \\
\hline \multirow[t]{5}{*}{ Escolarização } & Até ensino médio incompleto & 11 & 1,3 \\
\hline & Ensino médio completo & 213 & 23,7 \\
\hline & Ensino Superior incompleto & 158 & 17,6 \\
\hline & Ensino Superior completo & 155 & 17,2 \\
\hline & Pós-Graduação & 363 & 40,3 \\
\hline \multirow[t]{3}{*}{ Idade } & Abaixo de 34 anos & 309 & 34,3 \\
\hline & 34 a 40 & 280 & 31,1 \\
\hline & Acima de 40 & 311 & 34,6 \\
\hline \multirow[t]{2}{*}{ Condição na provisão } & Chefe & 340 & 37,8 \\
\hline & Contribui parcialmente & 539 & 59,9 \\
\hline \multirow[t]{2}{*}{ Tipo de vínculo } & Apenas um vínculo de emprego & 725 & 80,6 \\
\hline & Dois vínculos ou mais & 175 & 19,4 \\
\hline \multirow[t]{3}{*}{ Tempo de atuação } & Até 8 anos & 264 & 29,3 \\
\hline & 8 a 14 anos & 362 & 40,2 \\
\hline & Mais de 14 anos & 274 & 30,4 \\
\hline \multirow{3}{*}{$\begin{array}{l}\text { Local } \\
\text { de atendimento }\end{array}$} & Setores Fechados & 146 & 16,2 \\
\hline & Emergência & 82 & 9,1 \\
\hline & Outro & 672 & 74,7 \\
\hline
\end{tabular}

Tabela 2

Média e erro padrão da análise multivariada $(n=900)$

\begin{tabular}{lcc}
\hline Item & Média & Erro padrão \\
\hline \multicolumn{1}{c}{$\quad$ Vigor } & 5,00 & 1,69 \\
No meu trabalho, sinto-me cheio(a) de energia & 4,47 & 1,89 \\
No meu trabalho, sinto-me forte e com vigor & 4,84 & 2,01 \\
Quando acordo de manhã, sinto-me bem por ir trabalhar & & 1,85 \\
$\quad$ Dedicação & 4,68 & 2,00 \\
Estou entusiasmado com o meu trabalho & 4,74 & 1,58 \\
O meu trabalho inspira-me & 6,10 & \\
Tenho orgulho do trabalho que faço $\quad$ Absorção & & \\
Estou contente quando estou trabalhando intensamente & 4,48 & 2,05 \\
Estou imerso(a) no meu trabalho & 5,36 & 1,92 \\
Esqueço-me "do resto" quando estou trabalhando & 4,98 & 2,15 \\
\hline
\end{tabular}


evidenciada no item relacionado ao sentimento de força e vigor no trabalho. O teste de homogeneidade para as variâncias, por meio da estatística de Levene, resultou em um valor de 2,208, sendo não significativo $(\mathrm{t}=0,111)$, enquanto a Anova foi significativa $(0,014)$. Tais testes mostram que os grupos por idade podem ser comparados e a comparação entre eles apresenta diferenças significativas do nível de engajamento por idade.

A Análise Fatorial Exploratória confirmou que, nesta amostra, o engagement consistia numa variável unidimensional (apresentando uma carga fatorial entre 0,56 e 0,89 ). A Tabela 3 mostra as correlações apresentadas entre os 9 itens, todas significativas ao nível de $5 \%$, variando entre 0,356 a 0,788 .

O estudo realizado por Borges e colaboradores (2017) com 160 enfermeiros ( 80 da região do Porto e 80 em uma das ilhas dos Açores), observou-se que os enfermeiros do Porto e dos Açores apresentaram elevado nível de engagement, em que a dedicação foi o eixo com a média de maior índice. Para que se possa garantir ações seguras por parte da enfermagem são necessários níveis positivos de engagement desta categoria (Borges et al., 2017). O estudo realizado por Queirós, Borges e Abreu (2015), com 178 enfermeiros de hospitais do Porto em relação ao engagement, evidenciou elevado vigor, dedicação e absorção. Como argumentam Schaufelli e Bakker (2003), os indivíduos que apresentam baixos escores em vigor tem menos energia, enquanto os que apresentam altos índices possuem muita energia. Ainda para os autores, em relação à dedicação, aqueles que mostram escores elevados identificam-se intensamente com seu ambiente laboral devido à experiência ser desafiadora, significativa e inspiradora. Os profissionais que apresentam índices elevados para absorção são aqueles que estão intensamente envolvidos no trabalho ao ponto de não ver o tempo passar e com dificuldades em desapegar-se dele.

Os dados das estimativas demonstram que o engagement, visto como a média de seus nove atributos, não se relaciona de modo significativo com sexo $(\mathrm{p}=0,918, \mathrm{t}=0,103)$, escolarização $(\mathrm{p}=0,347, \mathrm{t}=-0,942)$, número de filhos $(\mathrm{p}=$ $0,885, \mathrm{t}=-0,144)$, vínculo exclusivo com hospital $(\mathrm{p}=$ $0,115, \mathrm{t}=-1,578)$, categoria funcional $(\mathrm{p}=0,144, \mathrm{~F}=1,808)$, relação familiar ( $\mathrm{p}=0,377, \mathrm{~F}=0,978)$, situação em termos

Tabela 3

Matriz de correlação entre os atributos do engagement

\begin{tabular}{|c|c|c|c|c|c|c|c|c|c|}
\hline & $\begin{array}{c}\text { No meu } \\
\text { trabalho, } \\
\text { sinto-me } \\
\text { cheio de } \\
\text { energia }\end{array}$ & $\begin{array}{c}\text { Estou } \\
\text { entusiasmado } \\
\text { com o meu } \\
\text { trabalho }\end{array}$ & $\begin{array}{c}\text { Estou } \\
\text { contente } \\
\text { quando estou } \\
\text { trabalhando } \\
\text { intensamente }\end{array}$ & $\begin{array}{c}\text { No meu } \\
\text { trabalho, } \\
\text { sinto-me } \\
\text { forte e } \\
\text { com vigor }\end{array}$ & $\begin{array}{c}\text { O meu } \\
\text { trabalho } \\
\text { inspira-me }\end{array}$ & $\begin{array}{c}\text { Estou } \\
\text { imerso(a) } \\
\text { no meu } \\
\text { trabalho }\end{array}$ & $\begin{array}{c}\text { Quando } \\
\text { acordo de } \\
\text { manhã, } \\
\text { sinto-me } \\
\text { bem por ir } \\
\text { trabalhar }\end{array}$ & $\begin{array}{c}\text { Tenho } \\
\text { orgulho } \\
\text { do } \\
\text { trabalho } \\
\text { que faço }\end{array}$ & $\begin{array}{l}\text { Esqueço-me } \\
\text { "do resto" } \\
\text { quando } \\
\text { estou } \\
\text { trabalhando }\end{array}$ \\
\hline $\begin{array}{l}\text { No meu trabalho, } \\
\text { sinto-me cheio(a) } \\
\text { de energia }\end{array}$ & 1 & $0,79^{* * *}$ & $0,69^{* *}$ & $0,74^{* *}$ & $0,62^{* *}$ & $0,49^{* * *}$ & $0,68^{* *}$ & $0,54^{* *}$ & $0,36^{* *}$ \\
\hline $\begin{array}{l}\text { Estou } \\
\text { entusiasmado } \\
\text { com o meu } \\
\text { trabalho }\end{array}$ & $0,79^{* *}$ & 1 & $0,73^{* *}$ & $0,78^{* *}$ & $0,74^{* *}$ & $0,52^{* *}$ & $0,70^{* *}$ & $0,54^{* *}$ & $0,40^{* *}$ \\
\hline $\begin{array}{l}\text { Estou contente } \\
\text { quando estou } \\
\text { trabalhando } \\
\text { intensamente }\end{array}$ & $0,69^{* *}$ & $0,73^{* *}$ & 1 & $0,76^{* *}$ & $0,65^{* *}$ & $0,47^{* *}$ & $0,66^{* *}$ & $0,51^{* *}$ & $0,38^{* *}$ \\
\hline $\begin{array}{l}\text { No meu trabalho, } \\
\text { sinto-me forte e } \\
\text { com vigor }\end{array}$ & $0,74^{* *}$ & $0,78^{* *}$ & $0,76^{* *}$ & 1 & $0,72^{* *}$ & $0,50^{* *}$ & $0,71^{* *}$ & $0,52^{* *}$ & $0,39^{* *}$ \\
\hline $\begin{array}{l}\text { O meu trabalho } \\
\text { inspira-me }\end{array}$ & $0,62^{* *}$ & $0,74^{* *}$ & $0,65^{* *}$ & $0,72^{* *}$ & 1 & $0,53^{* *}$ & $0,67^{* *}$ & $0,57^{* *}$ & $0,41^{* *}$ \\
\hline $\begin{array}{l}\text { Estou imerso(a) } \\
\text { no meu trabalho }\end{array}$ & $0,49^{* *}$ & $0,52^{* *}$ & $0,47^{* *}$ & $0,50^{* *}$ & $0,53^{* *}$ & 1 & $0,55^{* *}$ & $0,49^{* *}$ & $0,41^{* *}$ \\
\hline $\begin{array}{l}\text { Quando acordo } \\
\text { de manhã, sinto- } \\
\text { me bem por ir } \\
\text { trabalhar }\end{array}$ & $0,68^{* *}$ & $0,70^{* *}$ & $0,66^{* *}$ & $0,71^{* *}$ & $0,67^{* * *}$ & $0,55^{* *}$ & 1 & $0,55^{* *}$ & $0,42^{* *}$ \\
\hline $\begin{array}{l}\text { Tenho orgulho do } \\
\text { trabalho que faço }\end{array}$ & $0,54^{* *}$ & $0,54^{* *}$ & $0,51^{* *}$ & $0,52^{* *}$ & $0,57^{* *}$ & $0,49^{* *}$ & $0,55^{* *}$ & 1 & $0,46^{* *}$ \\
\hline $\begin{array}{l}\text { Esqueço-me "do } \\
\text { resto" quando } \\
\text { estou trabalhando }\end{array}$ & $0,36^{* *}$ & $0,40^{* * *}$ & $0,38^{* *}$ & $0,39^{* *}$ & $0,41^{* *}$ & $0,41^{* * *}$ & $0,42^{* *}$ & $0,46^{* *}$ & 1 \\
\hline
\end{tabular}

Nota: ** significante ao nivel de 0,01. 
de provisão da renda $(p=0,059, F=2,834)$, quantidade de vínculos de trabalho $(\mathrm{p}=0,022, \mathrm{~F}=3,830)$ e carga horária semanal $(\mathrm{p}=0,347, \mathrm{~F}=1,061)$. A única variável que demonstrou ser significativa foi a faixa etária (Tabela 4). Este resultado está de acordo com a ideia de que são as variáveis do contexto profissional as que podem ter maior importância para explicar o bem-estar dos trabalhadores, quando comparadas às variáveis individuais. A estatística de Levene para a homogeneidade das variâncias foi de 1,927 com significância de 0,146 , o que reforça a possibilidade de comparação entre grupos (Figura 1).
Há que se destacar, a esse respeito, que os recursos profissionais possam diminuir com a idade, os adultos mais velhos ainda podem experimentar espaços para crescimento em outros domínios da vida, como as relações sociais (Kooij et al., 2011). Mesmo com a força motora diminuindo com a idade, para os trabalhadores mais velhos, os motivos intrínsecos e de realização são mais significativos e importantes (Kooij et al., 2011). Cabe aos gestores, portanto, não somente a criação de oportunidades de treinamento, mas também, o estabelecimento de espaços de trabalho interessantes e que façam sentido para as

Tabela 4

Estatísticas de engagement para a idade

\begin{tabular}{lrr}
\hline Faixa etária & N & \multicolumn{1}{c}{ Subconjunto para alfa $=\mathbf{0 . 0 5}$} \\
\cline { 2 - 3 } Entre 34 a 40 anos & 280 & 4,75 \\
Abaixo de 34 anos & 309 & $\mathbf{2}$ \\
Acima de 40 anos & 311 & 5,05 \\
Sig. & & 5,07 \\
\hline
\end{tabular}

Nota: Teste Tukey HSD, são exibidas as médias para os grupos em subconjuntos homogêneos.

a. Usa o Tamanho da Amostra de Média Harmônica $=299,308$.

b. Os tamanhos de grupos são desiguais. A média harmônica dos tamanhos de grupos é usada. Os níveis de erro de Tipo I não são garantidos.

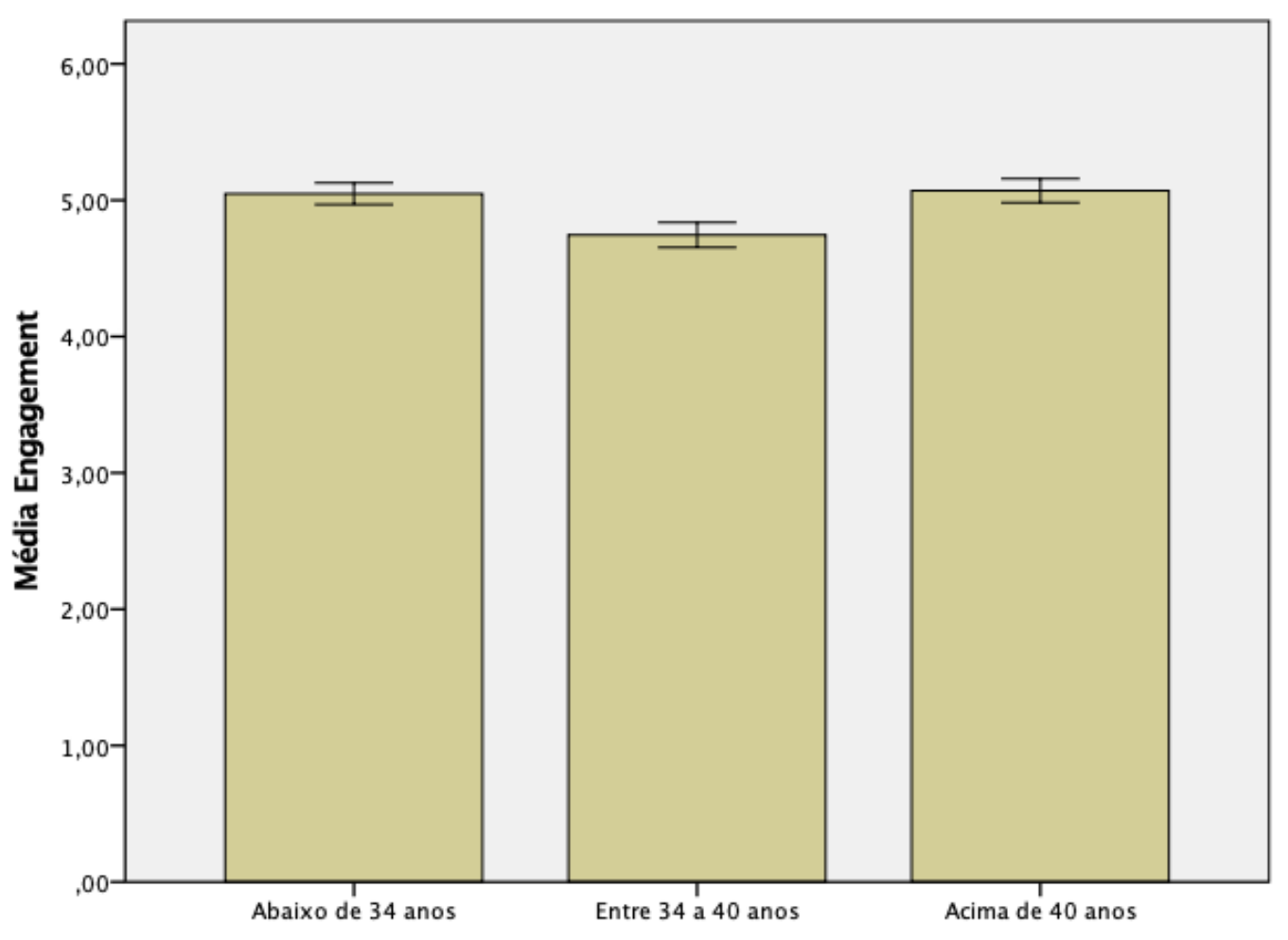

Faixa etária

Barras de erros: + /- $1 \mathrm{EP}$

Figura 1. Média do engagement para a faixa etária. 
vidas dos trabalhadores. Os adultos mais velhos veem suas experiências emocionais positivas perdurarem por mais tempo em comparação aos colegas mais jovens, pois são mais propensos a usar o otimismo como estratégia de regulação (Ng \& Feldman, 2010).

Nessa direção, o estudo de Avery, McKay e Wilson (2007) identificou que a relação entre a similaridade percebida para a idade entre os empregados mais velhos e seus pares e o engajamento no trabalho são mais fortes do que entre os trabalhadores mais jovens. O estudo realizado por Sousa, Pereira, Borges e Queirós (2015), com 323 enfermeiros de hospitais do Porto, evidenciou que o tempo de trabalho e a idade fazem diminuir a exaustão e a despersonalização, sendo que o tempo de trabalho eleva o vigor. No estudo de Borges e colaboradores (2017), a correlação entre o engajamento, a idade dos participantes e os anos de experiência profissional evidencia que entre os enfermeiros, o modelo demandas e recursos do trabalho favorece o engajamento, a despeito do afloramento da idade do profissional.

\section{CONCLUSÃO}

O presente estudo permitiu identificar características dos profissionais da enfermagem em relação ao engajamento em sua atividade laboral. Apesar de se saber que os enfermeiros são profissionais de risco em relação ao burnout, com este estudo foi possível observar que os enfermeiros exibem elevados níveis de engajamento, pois, mesmo com a desgastante rotina e as mais variadas dificuldades encontradas no ambiente laboral, a enfermagem é uma categoria que demonstra amor e comprometimento pela profissão, mostrando-se orgulhosa pela escolha profissional, independente de sexo, idade, tempo de serviço, entre outros. Tal conclusão é o que faz explicar os resultados desta pesquisa. A intensa relação afetiva e de dedicação que esses profissionais demonstraram ter pela profissão e seus pacientes parece contribuir de maneira significativa para a permanência na área da saúde e em suas instituições de trabalho.

Foi possível evidenciar que, apesar de as condições de trabalho por vezes adversas - como a falta de recursos humanos e materiais e excesso de carga de trabalho, que existe no cotidiano destes profissionais - os indivíduos mostraram-se com um nível moderado a intenso de engajamento. Não houve diferenças entre o gênero, o enquadramento profissional, como enfermeiro ou técnico de enfermagem, a escolarização, a condição na provisão familiar, o número de vínculos de trabalho, o tempo de atuação, a atividade em nível hospitalar ou não, assim como o setor em que atua.

Em relação à idade, observada por meio da faixa etária, ficou evidenciado que tanto os profissionais mais jovens quanto os profissionais mais "maduros" apresentaram níveis mais elevado de engajamento. Os profissionais entre $30 \mathrm{e}$ 40 anos foram aqueles que apresentaram menores níveis de engajamento. No caso da faixa etária mais elevada, tal resultado indica que esses profissionais ainda possuem muito a contribuir em seu meio laboral com sua experiência e otimismo.

A forma de acesso à população do estudo, incluindo a necessidade de manutenção de um período de tempo para o acesso ao instrumento, além das idiossincrasias deste grupo de profissionais, colocam-se como limitações do estudo.

Considerando-se que ainda há poucas pesquisas sobre engajamento, sugere-se o aprofundamento, em estudos futuros, das condições do ambiente de trabalho dos profissionais da enfermagem, uma vez que as variáveis organizacionais tendem a se sobrepor às variáveis individuais. No mesmo sentido, ciente das diferentes gerações presentes no ambiente de trabalho, há espaço para avaliar em que medida essa diversidade etária impacta nos recursos laborais oferecidos pelas organizações prestadores de serviços em saúde e, por consequência, no engajamento dos profissionais.

\section{REFERÊNCIAS}

Avery, D. R., Mckay, P. F., \& Wilson, D. C. (2007). Engaging the Aging Workforce: the relationship between perceived age similarity, satisfaction with coworkers, and employee engagement. Journal of Applied Psychology, 92(6), 15421556. https://doi.org/10.1037/0021-9010.92.6.1542

Barboza, J. I. R. A, Baresin, R. (2007). A síndrome de burnout em graduandos de enfermagem. Einstein, 5(3), 225-230.

Borges, E., Abreu, M., Queirós, C., \& Maio, T. (2017). Engagement em enfermeiros: estudo comparativo entre Portugal Continental e Açores. International Journal on Working Conditions, 14, 154-166.

Conselho Federal de Enfermagem - Cofen. (2018). Enfermagem em Números. Brasília, 2018. Disponível em: http://www.cofen. gov.br/enfermagem-em-numeros
Demerouti, E., Mostert, K. \& Bakker, A. B. (2010). Burnout and Work Engagement: A thorough investigation of the independency of both constructs. Journal of Occupational Health Psychology, 15(3), 209-222. Doi: 10.1037/a0019408.

Ferreira, N. N., \& Lucca, S. R. (2015). Síndrome de burnout em técnicos de enfermagem de um hospital público do Estado de São Paulo. Revista Brasileira de Epidemiologia, 18(1), 68-79. Doi: https://doi.org/10.1590/1980-5497201500010006.

França, F. M., Ferrari, R., Ferrari, D. C., \& Alves, E. D. (2012). Burnout e os aspectos laborais na equipe de enfermagem de dois hospitais de médio porte. Revista Latino-Americana de Enfermagem, 20(5), 9 telas.

Freitas, L. A. V., \& Charão-Brito, L. (2016). Engajamento no Trabalho: um estudo em profissionais multidisciplinares de 
saúde em um município da Região Sul. Revista CesumarCiências Humanas e Sociais Aplicadas, 21(2), 407-419.

Gonçalves, A. R., Galvão, A., Escanciano, S., Pinheiro, M., \& Gomes, M. J. (2018). Stress e engagement na profissão de enfermagem: análise de dois contextos internacionais. Revista Portuguesa de Enfermagem de Saúde Mental, (spe6), 59-64. Doi: 10.19131/rpesm.0214

Hanzelmann, R. S., \& Passos, J. P. (2010). Imagens e representações da enfermagem acerca do stress e sua influência na atividade laboral. Revista Escola Enfermagem USP, 44(3), 694-701.

International Labour Organization [ILO] (2019). Safety and Health at the Heart of the Future of Work: A compilation of think pieces. Switzerland: Author.

Kahn, W. A. (1990). Psychological Conditions of Personal Engagement and Disengagement at Work. The Academy of Management Journal, 33(4), 692-724.

Kooij, D. T., Lange, A. H., Jansen, P. G. W., Kanfer, R., \& Dikkers, J. S. E. (2011). Age and work-related motives: Results of a meta- analysis. Journal of Organization Behavior, 32, 197-225. Doi: $10.1002 /$ job.665

Martins, V., Costa, L. V., \& Siqueira, M. (2015). O impacto do comprometimento afetivo e do engajamento no trabalho sobre os comportamentos de cidadania organizacional. RACEFRevista de Administração, Contabilidade e Economia da Fundace, 6(2), 1-13.

Ng, T. W. H., \& Feldman, D. C. (2010). The relationships of age with job atitudes: a meta analysis. Personnel Psycology, 63,77-718. https://doi.org/10.1111/j.1744-6570.2010.01184.x

Obregon, S. L., Porto, A., Kochhan, S. C., Costa, V. M. F., CharãoBrito, L., \& Lopes, F. D. (2016). Engajamento no trabalho: Uma análise das publicações da última década. Espacios, 37(24), 15.

Pestana, M. H., \& Gageiro, J. N. (2000). Análise de dados para ciências sociais: a complementaridade do SPSS ( $2^{\mathrm{a}}$ ed.). Lisboa, Sílabo.

Pocinho, M.; \& Perestrelo, C. X. (2011). Um ensaio sobre burnout, engagement e estratégias de coping na profissão docente. Educação e Pesquisa, 37(3), 513-528.

Queirós, C., Borges, E., \& Abreu, M. (2015). Burnout e Engagement em enfermeiros portuenses. Anais do Congresso Internacional de Saúde Familiar \& Comunitária, 1, Encontro do Dia Internacional da família, 7, Coimbra, Portugal.

Rich, B. L., Lepine, J. A., \& Crawford, E. R. (2010). Job engagement: Antecedents and effects on job performance. Academy of Management Journal, 53(3), 617-635.

Rolim, C. S. S. (2013). Estresse e síndrome de Burnout em profissionais de enfermagem. Revista Brasileira de Pesquisa e Saúde, 15(3), 103-113.

Salas-Vallina, A., \& Alegre, J. (2017). The role of gender, age, job level and job security on physician's engagement in Spanish public hospitals. Universia Business Review, 56, 36-51. Doi: 10.3232/UBR.2017.V14.N4.02

Schaufeli, W., \& Bakker, A. (2003). Utrecht Work Engagement Scale. Preliminary Manual, Version 1.1.

Schaufeli, W. B., Bakker, A. B., \& Salanova, M. (2006). The measurement of work engagement with a short questionnaire: a cross-national study. Educational and Psychological Measurement, 66, 701-716.

Schaufeli, W. B., \& Taris, T. W. (2014). A Critical Review of the Job Demands-Resources Model: implications for improving work and health. In: G. F. Bauer \& O. Hämmig. O. Bridging Occupational, Organizational and Public Health: a transdisciplinary approach (pp. 43-68). Berlin: Springer Science \& Business Media, Berlin.

Silva, J. L. L., Soares, R. S., Costa, F. S., Ramos, D. S., Lima, F. B., \& Teixeira, L. R. (2015). Fatores psicossociais e prevalência da síndrome de Burnout entre trabalhadores de enfermagem intensivistas. Revista Brasileira Terapia Intensiva, 27(2), 125-133.

Sousa, L., Pereira, A. M., Borges, E., \& Queirós, C. (2015). Influência da Satisfação no trabalho e do Engagement no Burnout de Enfermeiros. Anais do Congresso Internacional de Saúde Familiar \& Comunitária, 1, Encontro do Dia Internacional da familia, 7. Coimbra, Portugal.

Vicencio-Ayub, E. V., Jurado-Cárdenas, S., \& Valencia-Cruz, A. (2015). Work engagement and occupational burnout: Its relation to organizational socialization and psychological resilience. Journal of Behavior, Health \& Social Issues, 6(2), 45-55. Doi: http://dx.doi.org/10.22201/fesi.20070780.2014.6.2.48591 